\title{
Accuracy of visual inspection with acetic acid for cervical pathology screening in low cost setup
}

\author{
Aditya Sisodia* \\ Department of Obstetrics and Gynecology, Govt Medical College Rajnandgaon, Chhattisgarh, India \\ Received: 10 February 2020 \\ Accepted: 18 February 2020 \\ *Correspondence: \\ Dr. Aditya Sisodia, \\ E-mail: sisodiakorba@gmail.com \\ Copyright: (C) the author(s), publisher and licensee Medip Academy. This is an open-access article distributed under \\ the terms of the Creative Commons Attribution Non-Commercial License, which permits unrestricted non-commercial \\ use, distribution, and reproduction in any medium, provided the original work is properly cited.
}

\begin{abstract}
Background: Cancer cervix, a preventable disease continues to be a cause of great concern to women's health, being associated with agonizing morbidity and high mortality. Approximately 493,100 new cases and more than 273,000 deaths occur each year, among women worldwide.IN India the screening is largely based on pap smear, which is cumbersome procedure due to transportation of samples and follow up of patients are not usually feasible. Visual inspection with Acetic acid (VIA) is simple and easy to teach procedure. Follow-up of patients usually doesn't require.

Methods: This study includes a total of 1000 patients who attended OPD of gynecology. Each patient is subjected to VIA examination. Biopsy is taken from women with abnormal findings on VIA. The objectives of this study were to evaluate the sensitivity and specificity and positive predictive value of inspection of cervix with acetic acid in precancerous lesions of cervix.

Results: When biopsy is taken as reference standard, VIA has sensitivity of $97.7 \%$, specificity of $76.6 \%$, PPV of $62.9 \%$ and NPV of $98.5 \%$. The accuracy rate for VIA is $84.8 \%$.

Conclusions: VIA is highly sensitive for diagnosis and treatment of cervical pathology at the same sitting. It can be done cost effectively in low resource set up.
\end{abstract}

Keywords: Biopsy, Invasive cancer of cervix, Preinvasive lesion of cervix, Pap Smear, Visual inspection with acetic acid

\section{INTRODUCTION}

India, which accounts for one sixth of world's population, also bears one fifth of world's burden of cervical cancer. ${ }^{1}$ It is the foremost common cancer among women in India. There are approximately 130,000 new cases of cervical cancer in India per annum and therefore the disease is reported to be liable for almost $20 \%$ of all female deaths.

Simultaneously, there's also evidence that India is on the verge of an outsized HIV epidemic. The Indian National AIDS Control Organization estimates that the number of people living with HIV is approximately 5.1 million (38\% of whom were women). This suggests cause for concern given the strong association between HIV and HPV infections and evidence of more rapid progression of HPV infections to cervical neoplasia in HIV infected women. ${ }^{1}$

Global evidence demonstrates that the key to reducing cervical cancer morbidity and mortality is early detection coupled with timely treatment of cervical precancerous lesions.

WHO states that cervical cancer is totally preventable disease. Preventable but not prevented is the glaring reality in India. Given this reality and the fact that screening by pap smear (cytological) does have pitfalls. 
Cytology based screening programmes for cervical cancer cannot be provided on a large scale in developing countries because they are based on transportation, communication, follow-up and training. It is apparent that cervical cancer screening based on an approach other than just pap smears is needed.

One such method is visual inspection with acetic acid. The cervix is washed with acetic acid and then inspected by eye for evidence of disease. This has potential advantages over traditional screening techniques in poorly resourced locations as it does not require a second person for interpretation of results nor a second visit by the patient to collect the report as there is immediate feedback of test results to the patient and importantly, treatment can be provided immediately after the test. ${ }^{2}$ VIA has been recommended by WHO as an alternative to cytology to pick up a patient at risk for cancer cervix. ${ }^{3}$

\section{Advantages of $\mathrm{VIA}$}

- Simple and easy to learn approach

- VIA is cheap so very cost effective

- The negative predictive value of VIA is sufficiently high to assure screening of negative women.

Most important advantage particularly in a population like that seen in the present study with high illiteracy rate and poor follow up is immediate availability of test result. This facilitates diagnosis (colposcopy/biopsy) and treatment to be carried out in the same visit. Single visit approach provides treatment with cryotherapy without opting for diagnostic investigations or referral. ${ }^{4}$ This approach has been found to be safe, acceptable and feasible.

The three types of errors leading to false-negative results of Papanicolaou smears like (1) sampling error (2) screening error (3) interpretative errors can be improved by using the Ayre spatula first followed by the endocervical brush. Fewer smears will be obscured by blood which could result in more squamous intraepithelial lesions being detected. The present study was undertaken to assess the sensitivity and specificity of VIA in picking up preinvasive or invasive cancer in an abnormal cervix.

Objective of this study were to evaluate the sensitivity and specificity and positive predictive value of inspection of cervix with acetic acid in precancerous lesions of cervix. To evaluate VIA of cervix in picking preinvasive and invasive cancer of cervix.

\section{METHODS}

This study was conducted at Govt. Medical College, Rajnandgaon over a period of 2 years, from September 2015 to August 2017. The patients for the study were selected randomly from those attending outpatient department of obstetrics and gynecology at Govt. Medical College, Rajnandgaon.

It is a prospective study conducted on 1000 gynecological outpatients for visual inspection of cervix after application of 3\% acetic acid followed by biopsy in women with abnormal or suspicious areas on VIA, in predicting sensitivity, specificity and positive predictive value in precancerous lesions of cervix.

\section{Inclusion criteria}

- Women aged 18 years and above and sexually active

- Multiparous women

- Women treated for vaginal infections

- Women with history of sexually transmitted diseases

- Women with family history of genital malignancies.

\section{Exclusion criteria}

- Pregnant women

- Women with post hysterectomy status

- Women with current vaginal infections

- Women with previous history of treatment for cervical carcinoma

- Women with active bleeding $\mathrm{p} / \mathrm{v}$.

\section{Materials}

- Sterile Sims speculum and anterior vaginal wall retractor

- $\quad$ Sponge holding forceps and swabs and cotton swabs on sticks

- Normal saline

- Acetic acid 3\% - freshly prepared. Light source

- Sterile Cusco's bivalve speculum of different standard sizes

- Cervical punch biopsy forceps.

- Examination table.

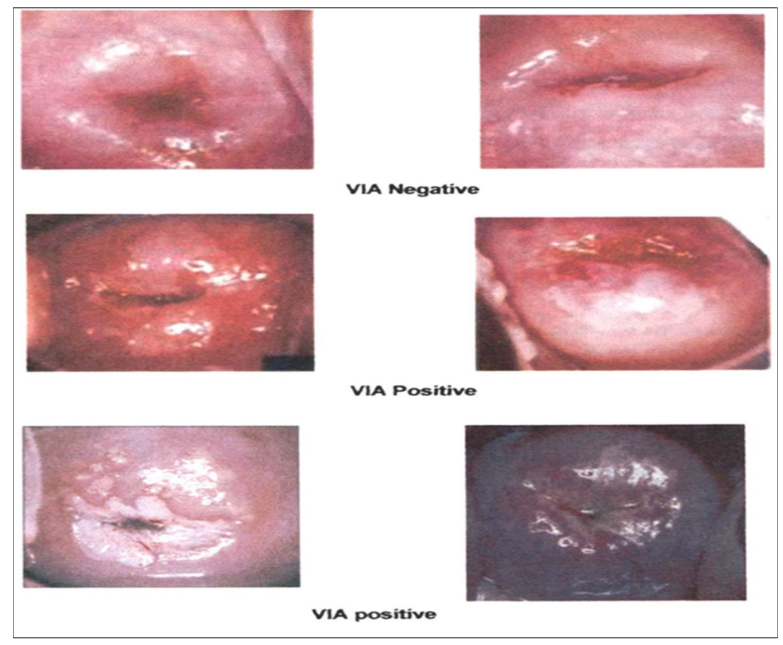

Figure 1: VIA positive and negative cases. 


\section{Method of collection of data}

After explaining the need for, and the procedure, after taking verbal consent, the data is collected by examination of the outpatients. Patient is put in lithotomy position. First an unaided visual inspection of cervix is performed under good illumination and findings noted. Cervix is painted with $3 \%$ freshly prepared acetic acid solution using a sterile cotton swab. Cervix is inspected after 1 minute and the changes in surface epithelium were noted. Results are noted either positive if there is acetowhite areas or negative if no acetowhite areas seen. The VIA findings are recorded. Direct punch biopsy is taken from those who showed positive VIA (Figure 1).

Biopsy is used as reference standard for statistical calculations. Biopsy is considered positive if it shows CIN lesions.

\section{RESULTS}

This is a prospective study undertaken to investigate the risk factors in association with premalignant and malignant lesions of cervix with visual inspection of cervix after application of 3\% acetic-acid and biopsy of abnormal lesions. The study consisted of 1000 gynaecological patients.

In our prospective study sensitivity of VIA is $97.2 \%$, specificity is $76.6 \%$, positive predictive value is $62.9 \%$, and negative predictive value is $98.5 \%$ (Table 1 ).
Table 1: Statistical analysis, comparison of via with biopsy.

\begin{tabular}{|l|l|ll|}
\hline $\begin{array}{l}\text { VIA } \\
\text { findings }\end{array}$ & $\begin{array}{l}\text { Total } \\
\text { number of } \\
\text { patients }\end{array}$ & Positive & Negative \\
\hline Positive & 448 & $282(62.9 \%)$ & $166(37.1 \%)$ \\
\hline Negative & 552 & $8(1.4 \%)$ & $544(98.6 \%)$ \\
\hline Total & 1000 & $290(29.0 \%)$ & $710(71.0 \%)$ \\
\hline Sensitivity \% & 97.2 & & \\
\hline Specificity $\%$ & 76.6 & & \\
\hline PPV \% & 62.9 & & \\
\hline NPV \% & 98.5 & \\
\hline Accuracy $\%$ & 84.8 & \\
\hline p value & $\mathrm{c}^{2}=218.215 ; \mathrm{p}<0.001 * *$ \\
\hline
\end{tabular}

\section{DISCUSSION}

The study was done on patients attending gynecological outpatient departments of obstetrics and gynaecology in Govt. Medical College Rajnadgaon Chhattisgarh. The study is compared with other authors who had done similar studies and results evaluated. In present study five hundred cases were subjected to VIA patients with acetowhite areas were subjected to biopsy.

In a study conducted by Sankarnarayanan sensitivity was $96 \%$ and $90 \%$ respectively. ${ }^{5}$

Goel et al, found sensitivity $96.7 \%$. Bharani et al found sensitivity of $100 \%{ }^{6,7}$

Table 2: Comparison of sensitivity of VIA of various studies.

\begin{tabular}{|c|c|c|c|c|}
\hline First author (year) & Country & No. of women & Sensitivity & Reference Standard \\
\hline Sankaranarayanan ${ }^{4}$ & India & 1351 & $96 \%$ & Colposcopy/Biopsy \\
\hline Sankaranarayanan et $\mathrm{al}^{5}$ & India & 2935 & $90 \%$ & Colposcopy/Biopsy \\
\hline Goel et $\mathrm{al}^{6}$ & India & - & $96.7 \%$ & Colposcopy/Biopsy \\
\hline Barani et al ${ }^{7}$ & India & - & $100 \%$ & Colposcopy/Biopsy \\
\hline Present study & India & 1000 & $97.7 \%$ & Biopsy \\
\hline
\end{tabular}

Table 3: Comparison of specificity of VIA of various authors.

\begin{tabular}{|lll|}
\hline First author (year) & Specificity & Reference Standard \\
\hline Doh et al & & Colposcopy/Biopsy \\
\hline Sankaranarayanan et al & $77.6 \%$ & Colposcopy/Biopsy \\
\hline Sankaranarayanan et al & $81.9 \%$ & Colposcopy/Biopsy \\
\hline Present study & $65 \%$ & Biopsy \\
\hline
\end{tabular}

In present study sensitivity was $97.7 \%$ which was correlating with Goel et al6 and Sankarnarayanan Table 2.4

In the study conducted by Doh et al specificity was $77.6 \%{ }^{8}$
In a study by Sankarnarayanan et al, specificity was $65 \%$ and $81.9 \%$ respectively.

In the present study specificity is $76.6 \%$ which correlated with study conducted by Doh et al and Sankarnarayanan et al Table 3. 
In a study by Barani et al, positive predictive value was $75 \%$.

In a study conducted by Megevand et al positive predictive value was $72 \%$. In our prospective study positive predictive value is $62.9 \%$ which corresponds to above mention study done previously Table 4 .

Table 4: Comparison of positive predictive value of VIA of various authors.

\begin{tabular}{|lll|}
\hline First author & $\begin{array}{l}\text { Positive } \\
\text { predictive value }\end{array}$ & $\begin{array}{l}\text { Reference } \\
\text { standard }\end{array}$ \\
\hline Barani et al & $75 \%$ & $\begin{array}{l}\text { Colposcopy } \\
\text { /Biopsy }\end{array}$ \\
\hline Megevand et al, ${ }^{9}$ & $72 \%$ & $\begin{array}{l}\text { Colposcopy } \\
\text { Biopsy }\end{array}$ \\
\hline Present study & $62.9 \%$ & Biopsy \\
\hline
\end{tabular}

\section{CONCLUSION}

During the past decade, much has been written about the challenges involved in detecting cervical cancer in lowresource settings and the strategies that are likely to be most effective in these settings.

The detection of intraepithelial or preclinical invasive cervical neoplasia should not depend on the possession of colposcope. On the other hand, use of colposcopy is essential for the selection of CIN that can be treated with ultraconservative therapy or with colposcopically directed conisation.

Many aspects of VIA make it an appealing approach for use in low-resource settings. In most cases, costs associated with launching and sustaining VIA screening are lower than those associated with other methods. VIA is a relatively simple, easy-to-learn approach that is only somewhat reliant upon infrastructure for its adequate performance, assuming that sufficiently trained providers are available. The approach does not require laboratory involvement and non-physicians can perform the procedure, provided that they receive adequate and ongoing training. As a result, VIA generally has the potential for greater population coverage than other available screening approaches. The results of the procedure are available immediately, making it possible to provide further management, including an offer of immediate treatment of some suspected precancerous lesions during the same visit.

Hence, the benefits of low-cost, ease of implementation and a point-of-care diagnosis and treatment algorithm will be powerful incentives for developing countries to pursue visual inspection as a screening procedure for cervical cancer.

Funding: No funding sources Conflict of interest: None declared

Ethical approval: The study was approved by the Institutional Ethics Committee

\section{REFERENCES}

1. Sehgal A, Singh V, Bhambhani S, Luthra UK. Screening for cervical cancer by direct inspection. Lancet. 1991;338:282.

2. JHPIEGO Cervical Cancer Project. Visual inspection with acetic acid for cervical-cancer screening: qualities in a primary care setting. Lancet. 1999;353:869-73.

3. Belinson JL, Pretorius RG, Zhang WH, Wu LY, Qiao YL, Elson P. Cervical cancer screening by simple visual inspection after acetic acid. Obstet Gynecol. 2001;98(3):441-4.

4. Sankaranarayanan R, Wesley R, Somanathan T, Dhakad N, Shyamalakumary B, Amma NS, et al. Visual inspection of the uterine cervix after the application of acetic acid in the detection of cervical carcinoma and its precursors. Am Cancer Soc. 1998;83:2150-6.

5. Sankaranarayanan R, Budukh AM, Rajkumar R. Effective screening programmes for cervical cancer in low-and middle-income developing countries. Bull World Health Organ. 2001;79:954-62.

6. Goel A, Gandhi G, Batra S, Bhambhani S, Zutshi V, Sachdeva P. Visual inspection of the cervix with acetic acid for cervical intraepithelial lesions. Inter $\mathrm{J}$ Gynecol Obstet. 2005;88(1):25-30.

7. Nalliah S, Karikalan B, Kademane K. Multifaceted usage of HPV related tests and products in the management of cervical cancer: a review. Asian Pac J Cancer Prev. 2015;16(6):2145-50.

8. McCarey C, Pirek D, Tebeu PM, Boulvain M, Doh AS, Petignat P. Awareness of HPV and cervical cancer prevention among Cameroonian healthcare workers. BMC Women's Health. 2011;11(1):45.

9. Megevand E, Denny L, Dehaeck K, Soeters R, Bloch B. Acetic acid visualization of the cervix: an alternative to cytologic screening. Obstet Gynecol. 1996;88(3):383-6.

Cite this article as: Sisodia A. Accuracy of visual inspection with acetic acid for cervical pathology screening in low cost setup. Int J Reprod Contracept Obstet Gynecol 2020;9:1335-8. 